\title{
Clinical, Laboratory and Radiological Predictors of Extension of Oligoarticular Juvenile Idiopathic Arthritis: A Prospective Study
}

\author{
Hisham M. Habib ${ }^{\mathrm{a}}$, Dalia A Shaheen ${ }^{\mathrm{b}}$, Ghada O. Elsedfy, d
}

\begin{abstract}
Background: Juvenile rheumatoid arthritis (JRA) is the most common chronic rheumatic illness in children and is a significant cause of both short- and long-term disabilities. The aim of this study is to detect clinical, laboratory and radiological predictors of oligoarticular juvenile idiopathic arthritis (JIA), which could be used to identify children whose disease is likely to extend to a more severe phenotype.
\end{abstract}

\begin{abstract}
Methods: This study included 40 oligoarticular JIA patients with no more than two years disease duration. Patients were divided into 2 groups after 6 months of illness into persistent and extended phenotypes. All patients were subjected to clinical, laboratory and conventional radiological assessment.
\end{abstract}

Results: In extended JIA patients there was a significant increase in age of patients, upper limb joint involvement, bilateral symmetrical arthritis, disease activity and functional outcome measures. Moreover, a significant elevation of ESR and CRP levels and RF positivity, as well as ANA negativity and radiological findings of joint inflammation were evident in extended phenotype.

Conclusions: Many clinical, laboratory and radiological predictors of conversion to extended oligoarticular JIA patients were close to charactertics of polyarticular form of the disease.

Keywords: Juvenile rheumatoid arthritis; OligoarticularJIA; Extended oligoarticularJIA

\footnotetext{
Manuscript accepted for publication June 11, 2012

${ }^{a}$ Department of Rheumatology and Rehabilitation, Mansoura University, Egypt

${ }^{b}$ Department of Medical Biochemistry, Mansoura University, Egypt

${ }^{\mathrm{c}}$ Department of Pediatrics, Assiut University, Egypt

${ }^{\mathrm{d}}$ Corresponding author: Ghada O. Elsedfy, Department of Pediatrics,

Assiut University, Assiut, Egypt 71515. Email: gelsedfy@hotmail.com
}

doi:10.4021/ijcp29w

\section{Introduction}

Juvenile rheumatoid arthritis (JRA) is the most common chronic rheumatic illness in children and is a significant cause of both short- and long-term disabilities [1]. It is comprised of a heterogeneous group of several disease subtypes that are characterized by the onset of arthritis before the age of 16 years and has symptoms lasting at least 6 weeks [2].

Classification of JIA is an ongoing process and up till now has been predominantly based on clinical manifestations-mainly number of joints at onset of disease [3, 4]. International League of Associations for Rheumatology (ILAR) Classification of JIA had classified JIA into 7 categories. Oligoartritis form of disease is classified as arthritis affect 4 or more joints during first 6 months of disease. It has 2 subcategories; persistent oligoarthritis with affection no more than 4 joints throughout the disease course and extended oligoarthritis with affection more than 4 joints after the first 6 months of disease [5].

Although oligoarticular JIA may sometimes thought of as a benign condition, in fact, it leads to a wide spectrum of outcomes ranging from complete remission after discontinuation of medication to development of a severe extended form of JIA that spreads to involve many joints ( $\geq 5$ joints). Extended oligoarticular JIA can be highly erosive and destructive and may be difficult to control with conventional disease-modifying antirheumatic drugs, thus requiring longterm treatment. When oligoarticular JIA remains limited to $\leq$ 4 joints, so-called persistent oligoarticular JIA, it is typically relatively easy to control $[6,7]$. It is stated that up to $50 \%$ of oligoarticular patients develop extended disease, and 30\% will do so in the first 2 years after diagnosis [8]. Antinuclear antibody may be an important marker of disease activity in patients with JIA [9]. All patients with ANA-positive result should be grouped as a separate category in the classification of juvenile idiopathic arthritis [10].

The aim of this prospective study was to identify clinical, laboratory and radiological pridectors in children with oligoarticular juvenile idiopathic arthritis (JIA), which could be used to identify children whose disease is likely to extend to a more severe phenotype. 


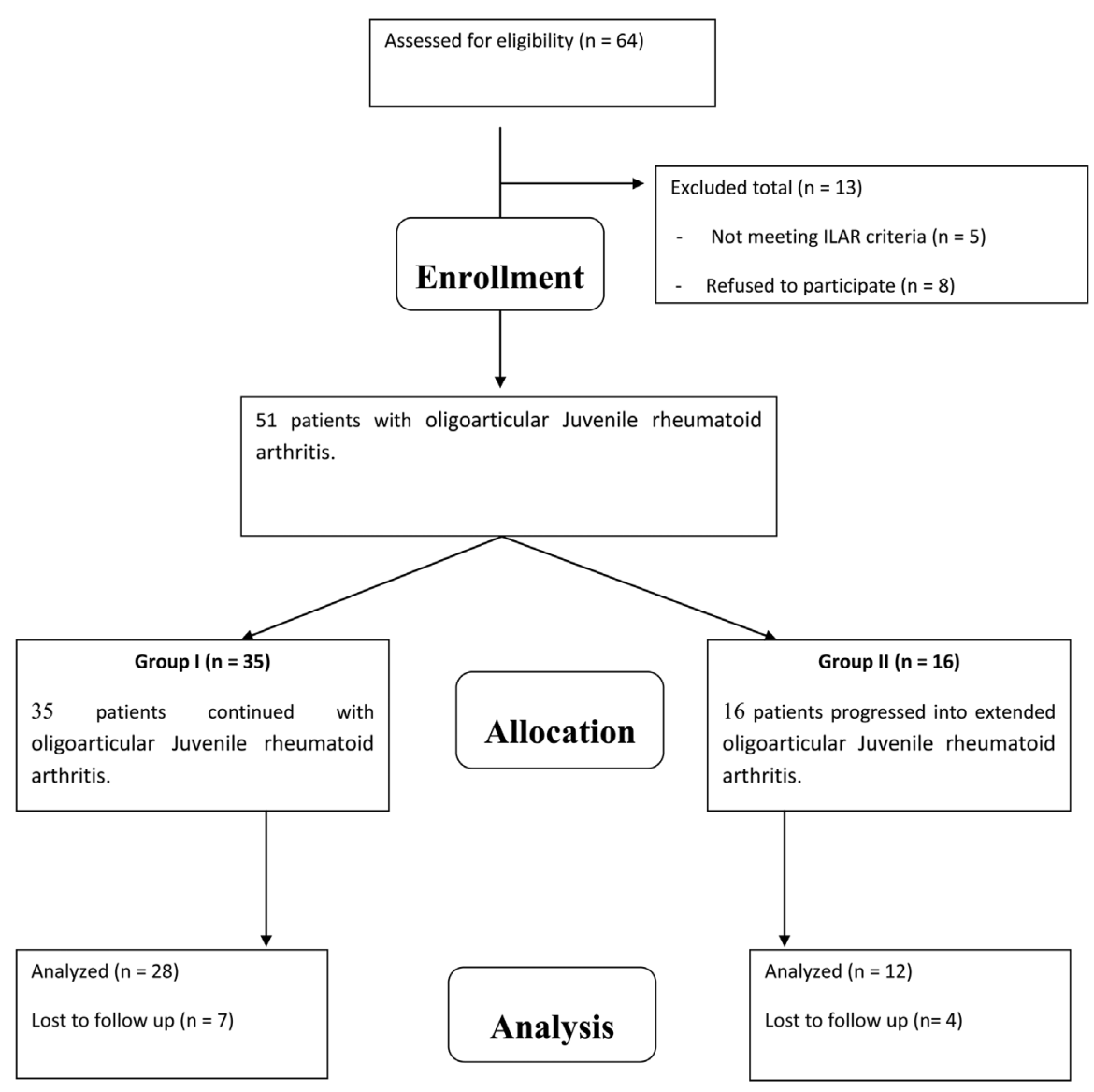

Figure 1. A flowchart of study procedure including patients' enrollment, allocation, follow up and analysis.

\section{Patients and Methods}

Figure 1 is a flowchart of study procedure including patients' enrollment, allocation, follow up and analysis. A total of 40 children ( 29 female and 11 male) with mean age of 9.3 years (range of 2.5 - 14 years) who met the International League of Associations for Rheumatology (ILAR) criteria for oligoarticular JIA [5] and were in the first 24 months of their disease duration were enrolled for this study. At that time, patients were divided into two groups according to the number of joint involved by inflammation (number of arthritic joints) after 6 months of base line diagnosis into two groups. Group "A" included 28 patients with persistent oligoarticular JIA, and group "B" included 12 patients with extended oligoarticular JIA. Group "A" comprised 20 female and 8 males with mean age was 7.2 years (range of 2.5 - 10.3 years). Group "B" comprised 8 females and 4 males with mean age were 10.5 years (range of $5.6-14$ years).

The study was approved by the hospital ethical committee and a written informed consent was taken from all patients' guardians who agreed to contribute in the study with full information about the study and procedures may request to do.
All patients were subjected to full history taking (either patient or parent if inconvenient), clinical examination with stress of the locomotors system, and the following measurements were recorded: Ritchie articular index score for assessing joint tenderness [11]. Functional capacity of the patients was assessed using the Steinbrocker grading system [12] and the Childhood Health Assessment Questionnaire (CHAQ) [13]. Clinical assessment of disease activity was performed using Visual analog scale $(0-10 \mathrm{~cm})$. Laboratory investigations were performed for all patients, including complete blood count, erythrocyte sedimentation rate by Wintergreens method, C-reactive protein detection by the latex agglutination slide test, and rheumatoid factor, antinuclear antibodies (ANA) and ophthalmological slit lamp examination for ANA positive patients. Conventional radiological examination of the arthritic joints in the anteroposterior and lateral views were done.

\section{Laboratory investigations}

Five milliliters of venous blood was collected from each subject and divided into three samples. The first one was used 
Table 1. Demographic and Clinical characteristics of the study population

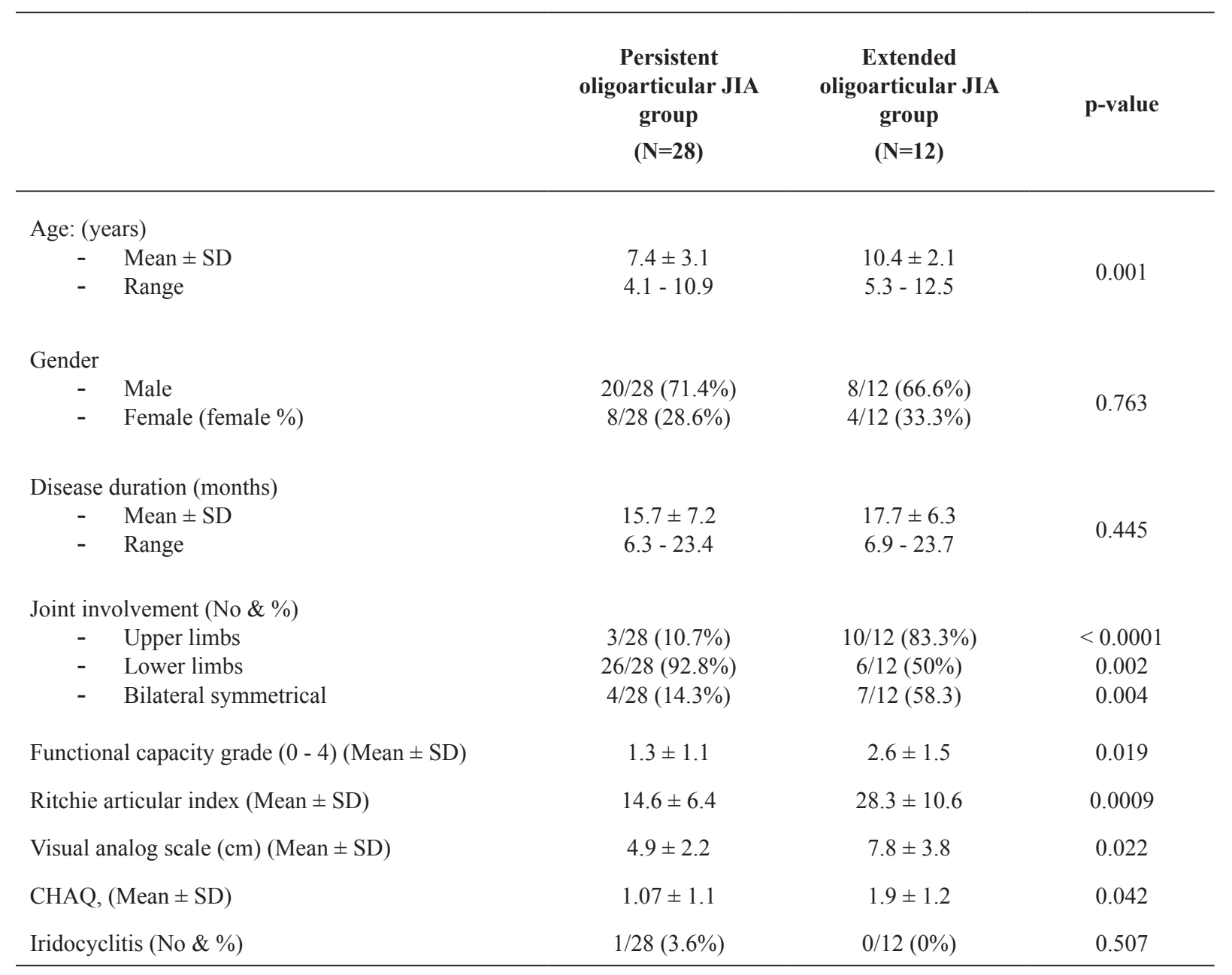

Abbreviations: CHAQ, Childhood Health Assessment Questionnaire (CHAQ)

for complete blood count, the second one for erythrocyte sedimentation rate by Westergren method. The third blood sample was used for quantitative determination of antinuclear antibodies (IgG, IgA, IgM), quantitative determination of $\mathrm{RF}$ and C-Reactive protein.

Human serum levels of ANA were measured using ELISA (IMTEC-ANA screen ITC 60001) (HUMAN-GERMANY). The readings were measured on an ELISA reader (HumaReader HS) at 450nm [14].

CRP and Serum human levels of RF were measured turbidimetrically at $552 \mathrm{~nm}$, and at $583 \mathrm{~nm}$ respectively using COBAS INTEGTRA 400 machine; Roche Diagnostics, Indianapolis, Ind) $[15,16]$.

\section{Statistical analysis}

Data were statistically described in terms of mean and standard deviation ( \pm SD). Comparison of the studied groups was done using the student's t test. Chi square test was uses to compare categorical variables. A p-value less than 0.05 was considered statistically significant. All statistical calculations were performed using computer programs Microsoft Excel version 7 (Microsoft Corporation, NY, USA) and SPSS version 15 (Statistical Package for the Social Science; SPSS Inc., Chicago, IL, USA) statistical program.

\section{Results}

In our study, twelve patients (30\%) out of original forty oligoarticular JIA patients changed in two years duration to extended oligoarticular JIA. All patients expressed this change after 6 months of their classification to oligoarticular JIA.

The clinical measures showed that in extended oligoarticular JIA patients, there is a statistically significant higher age group, upper limb joint involvement, bilateral symmetrical 
Table 2. Laboratory and radiological features of the study population

\begin{tabular}{|c|c|c|c|}
\hline & $\begin{array}{l}\text { Persistent } \\
\text { oligoarticular } \\
\text { JIA group } \\
(\mathbf{N}=\mathbf{2 8})\end{array}$ & $\begin{array}{l}\text { Extended } \\
\text { oligoarticular } \\
\text { JIA group } \\
(\mathrm{N}=12)\end{array}$ & p-value \\
\hline ESR level (mm/hour), (Mean \pm SD) & $33.9 \pm 12.1$ & $55.6 \pm 19.7$ & 0.003 \\
\hline CRP level $(\mathrm{mg} / \mathrm{L}),($ Mean $\pm \mathrm{SD})$ & $9.1 \pm 4.5$ & $22.3 \pm 10.6$ & 0.001 \\
\hline Rheumatoid factor positivity (No \& \%) & $0 / 28(0 \%)$ & $7 / 12(58.3)$ & $<0.0001$ \\
\hline Anemia $(\mathrm{Hb}<11$ gm \%) (No \& \%) & $12 / 28(42.9 \%)$ & $4 / 12(33.3 \%)$ & 0.573 \\
\hline ANA positivity (No \& \%) & $12 / 28(42.9 \%)$ & $1 / 12(8.3 \%)$ & 0.033 \\
\hline Radiological changes mainly JAO (No \& \%) & $5 / 28(17.9 \%)$ & $6 / 12(50 \%)$ & 0.037 \\
\hline
\end{tabular}

Abbreviations: ESR, erythrocyte sedimentation rate; CRP, C-reactive protein; ANA, antineuclear antibody; JAO, juxtaarticular osteoprosis

arthritis ( $\mathrm{p}<0.05$ in all). There is a statistically significant in lower limb joint involvement in persistent oligoarticular JIA patients. Disease activity and functional outcomes measures showed that there is a statistically significant activity and functional limitations in extended oligoarticular JIA patients compared to persistent oligoarticular JIA patients in all indices and measures applied ( $p<0.05$ in all). This was applied for Ritchie articular index score, functional capacity of the patients as assessed using the Steinbrocker grading system and the CHAQ and clinical assessment of disease activity as performed using Visual Analog Scale. There was no statistically significant difference between both group of patients in gender, disease duration, and occurrence of iridocyclitis (Table 1).

The laboratory measures showed that in extended oligoarticular JIA patients there is a statistically significant higher ESR level, CRP level, Rheumatoid factor positivity. There is no statistically significant difference between both group of patients in anaemia presence. There is statistically significant positive ANA in persistent oligoarticular JIA patients ( $\mathrm{p}$ $=0.033$ ) (Table 2).

The conventional radiological assessment showed that in extended oligoarticular JIA patients there is a statistically significant radiological changes of joint inflammation mainly of juxtaarticular osteoporosis character without erosion or joint space narrowing $(\mathrm{p}=0.037)$ (Table 2$)$.

\section{Discussion}

This study was performed to identify predictors of the future worsening and extension of the disease in children with an initially benign arthritis of oligoarticular JIA. This was done by screening of clinical, laboratory and conventional $\mathrm{x}$ ray parameters in such patients as early as the first 2 years of the onset of their illness giving the chance of the disease to express differentiation and study the behavior of the patients. Our study used some previously reported predictors of the extension to a more aggressive phenotype, as well as novel factors whose description in JIA are not yet clearly defined.

Our results revealed that extension of the disease to $\geq 5$ joints in this form of childhood arthritis has been reported to occur in as high as $30 \%$ of patients at 2 years and carries a far higher risk of chronic disability. Several clinical factors have been proposed as predictors of extension, such as higher age group, upper limb joint involvement, and bilateral symmetrical arthritis with higher chance for lower limb joint involvement in persistent phenotype. Furthermore, we have reported that disease activity and functional limitations measures were evident in extended form of the disease. Laboratory predictors of extension include high ESR level, CRP level, and rheumatoid factor positivity. We have found that extended JIA patients express radiological changes of inflammatory character of adult RA like juxtaarticular osteoporosis, joint erosion and joint space narrowing as early as the first 2 years of their illness.

Clinical reports investigating predictors of extension to more aggressive form in oligoarticular JIA patients were scant. Currently, there is no simple clinical algorithm can be applied to predict extension, but meeting this need would represent a major step forward in the care of children with 
arthritis. Our results were comparable to other reports. AlMatar et al -in their study- reported that extension of the disease to $\geq 5$ joints in this form of childhood arthritis has been reported to occur in as high as $50 \%$ of patients at 5 years and carries a far higher risk of chronic disability. Several clinical factors have been proposed as predictors of extension, such as a high erythrocyte sedimentation rate (ESR), upperlimb involvement, and involvement of $>1$ joint or symmetric joint involvement at presentation [17]. Moreover, Ravelli and Marini in summary of their study reported that greater severity/extension of arthritis at onset, symmetric disease, precocious hip/wrist involvement, the presence of rheumatoid factor, and prolonged active disease were the best predictors of a poor outcome. They concluded that joint symmetry and a higher erythrocyte sedimentation rate at onset were associated with a more severe course in oligoarticular JIA and prediction of long-term outcome in the first few months remains difficult [18]. Hence, it was wise thinking that we give a 24 month chance for our cohort to give chance for disease to express itself in a clearer pathway.

According to our results, it is evident that the presentation of "extended-to-be group" oligoarticular JIA patients behaves like that of polyarticular JIA phenotype. This can be understandable if this is linked to the immunopathogensis of both subtypes which will be presented in the synovial membrane. A significant difference in the CD3 T-cell population between the extended-to-be and persistent group was found. CD4 expression was significantly higher in the poly and extended-to-be oligo groups, again the extended-to-be group had more CD4 T cells than the persistent group. A marked B-cell infiltrates were more in the polyarticular group and were significantly higher in the extended-to-be group compared with the persistent group. A pronounced vascularisation was more in the polyarticular and extended-to-be oligoarticular groups, the extended-to-be group had significantly more vascularisation than the persistent group [19]. Moreover, investigations of immunophenotypes provide insights into adaptive immunity seems to have a prominent role in both polyarticular and oligoarticular JIA, and the morelimited arthritis observed in persistent oligoarticular JIA as compared with extended oligoarticular JIA. This may reflect more-potent immunoregulatory T-cell activity in the persistent oligoarticular JIA. Tumor necrosis factor seems to be a key mediator of both polyarticular and oligoarticular JIA, especially in the extended oligoarticular subtype, although elevated levels of other cytokines are also observed [20].

\section{Conclusion}

Our effort was directed to detect what characteristics in oligoarticular JIA patients make them to behave a more aggressive extended form of their illness as early as the first 2 years of their disease. Our findings denoted that these patients' characteristics were near to that of polyarticular form of JIA.
This goes in harmony with the fact that these patients will complete their illness like that of their polyarticular counterpart.

\section{Disclosure}

None.

\section{References}

1. Hahn YS, Kim JG. Pathogenesis and clinical manifestations of juvenile rheumatoid arthritis. Korean J Pediatr. 2010;53(11):921-930.

2. Kim KH, Kim DS. Juvenile idiopathic arthritis: Diagnosis and differential diagnosis. Korean J Pediatr. 2010;53(11):931-935.

3. Cimaz R, Moretti D, Pagnini I, Marino A, Cantarini L, Simonini G. What do cytokine profiles tell us about subsets of juvenile idiopathic arthritis? Curr Rheumatol Rep. 2012;14(2):150-154.

4. Minden K, Niewerth M. [Juvenile idiopathic arthritis-clinical subgroups and classification]. Z Rheumatol. 2008;67(2):100, 102-106, 108-110.

5. Petty RE, Southwood TR, Manners P, Baum J, Glass DN, Goldenberg J, He X, et al. International League of Associations for Rheumatology classification of juvenile idiopathic arthritis: second revision, Edmonton, 2001. J Rheumatol. 2004;31(2):390-392.

6. Packham JC, Hall MA. Long-term follow-up of 246 adults with juvenile idiopathic arthritis: functional outcome. Rheumatology (Oxford). 2002;41(12):14281435.

7. Nistala K, Woo P, Wedderburn LR. Juvenile idiopathic arthritis. In: Firestein GS, Budd RC, Harris ED, McInnes IB, Ruddy S, Sergent JS, editors. Kelley's textbook of rheumatology. 8th ed. Philadelphia: Saunders Elsevier; 2008. pp. 1657-76

8. Guillaume S, Prieur AM, Coste J, Job-Deslandre C. Long-term outcome and prognosis in oligoarticularonset juvenile idiopathic arthritis. Arthritis Rheum. 2000;43(8):1858-1865.

9. Shin JI, Kim KH, Chun JK, Lee TJ, Kim KJ, Kim HS, Kim DS. Prevalence and patterns of anti-nuclear antibodies in Korean children with juvenile idiopathic arthritis according to ILAR criteria. Scand J Rheumatol. 2008;37(5):348-351.

10. Ravelli A, Varnier GC, Oliveira S, Castell E, Arguedas O, Magnani A, Pistorio A, et al. Antinuclear antibodypositive patients should be grouped as a separate category in the classification of juvenile idiopathic arthritis. Arthritis Rheum. 2011;63(1):267-275.

11. Ritchie DM, Boyle JA, McInnes JM, Jasani MK, Dala- 
kos TG, Grieveson P, Buchanan WW. Clinical studies with an articular index for the assessment of joint tenderness in patients with rheumatoid arthritis. Q J Med. 1968;37(147):393-406.

12. Steinbrocker O, Trager GH, Butterman RC. Theraputic criteria in juvenile rheumatoid arthritis. JAMA. 1949; 140:659-62

13. Singh G, Athreya BH, Fries JF, Goldsmith DP. Measurement of health status in children with juvenile rheumatoid arthritis. Arthritis Rheum. 1994;37(12):1761-1769.

14. Muro Y. Antinuclear antibodies. Autoimmunity. 2005;38(1):3-9.

15. Sager D.Wernick RM, Davey MP. Assays for Rheumatoid factor: a review of their utility and limitations in clinical practice .Lab Med .1992; 23:15-18.

16. Eda S, Kaufmann J, Roos W, Pohl S. Development of a new microparticle-enhanced turbidimetric assay for C-reactive protein with superior features in ana- lytical sensitivity and dynamic range. J Clin Lab Anal. 1998;12(3):137-144.

17. Al-Matar MJ, Petty RE, Tucker LB, Malleson PN, Schroeder ML, Cabral DA. The early pattern of joint involvement predicts disease progression in children with oligoarticular (pauciarticular) juvenile rheumatoid arthritis. Arthritis Rheum. 2002;46(10):2708-2715.

18. Ravelli A, Martini A. Early predictors of outcome in juvenile idiopathic arthritis. Clin Exp Rheumatol. 2003;21(5 Suppl 31):S89-93.

19. Finnegan S, Clarke S, Gibson D, McAllister C, Rooney M. Synovial membrane immunohistology in early untreated juvenile idiopathic arthritis: differences between clinical subgroups. Ann Rheum Dis. 2011;70(10):18421850.

20. Macaubas C, Nguyen K, Milojevic D, Park JL, Mellins ED. Oligoarticular and polyarticular JIA: epidemiology and pathogenesis. Nat Rev Rheumatol. 2009;5(11):616-626. 